\title{
Genetic diversity of rotavirus genome segment 6 (encoding VP6) in Pretoria, South Africa
}

\author{
Martin M Nyaga ${ }^{1 *}$, Mathew D Esona ${ }^{1,2}$, Khuzwayo C Jere ${ }^{1,3}$, Ina Peenze ${ }^{1}$, Mapaseka L Seheri ${ }^{1}$ \\ and M Jeffrey Mphahlele ${ }^{1}$
}

\begin{abstract}
Background: Rotavirus viral protein 6 (VP6), encoded by genome segment (GS) 6, is the primary target for rotavirus diagnosis by serological and some molecular techniques. Selected full length nucleotide sequences of GS 6 of rotavirus strains from South Africa were sequenced and analysed to determine genetic diversity and variations within the circulating rotaviruses.

Findings: The VP6 amplicons were sequenced using the Sanger ABI 3130xl. Phylogenetic and pairwise analysis revealed that the VP6 genes of the study strains belonged to two different VP6 [I] genotypes. Five sequences were assigned genotype 11 and seven as genotype 12. Comparison of the group specific antigenic regions of the South African strains to the reference strains, shows that the South African VP6 sequences belonging to the VP6 genotype 12 were highly conserved, with only two amino acids changes at positions 239 (T>N) and $261(\mid>V)$. On the other hand, South African VP6 sequences belonging to 11 genotypes revealed several amino acid variations mostly within the antigenic region III.

Conclusions: Rotavirus strains with 11 and 12 genotype are predominantly circulating within the South African communities of which the later seems to be more conserved within the antigenic regions. The observed genetic variations observed within GS 6 of rotaviruses analysed in the current study are unlikely to impact negatively on the performance of the current VP6-based detection methods. Nevertheless, investigators should continually consider this diversity and adapt the primer design for the detection and characterization of the VP6 gene accordingly.
\end{abstract}

Keywords: Rotaviruses; Viral protein 6; Genetic diversity; South Africa; Pretoria

\section{Findings Introduction}

Rotaviruses form a genus rotavirus within the Reoviridae family (Estes and Kapikian 2007). Group A rotaviruses (RVA) are the major cause of severe dehydrating diarrhoea. Every year RVA infects approximately 114 million children that leads to 453,000 childhood deaths of which most occur in Africa and Asia (Tate et al. 2012). In South Africa, approximately 17,644 to 25,630 children are hospitalised of which 2,882 die annually due to rotavirus diarrhoea (WHO, 2012). An estimated 224 to 318 children die due to rotavirus disease in Pretoria and neighboring Brits areas of South Africa (Mapaseka et al. 2010).

\footnotetext{
* Correspondence: wamodylan@gmail.com

${ }^{1}$ Medical Research Council/Diarrhoeal Pathogens Research Unit, Department of Virology, Medunsa Campus, University of Limpopo/NHLS Dr George Mukhari Tertiary Laboratory, Pretoria, South Africa

Full list of author information is available at the end of the article
}

RVA are non-enveloped tripled-layered enteric viruses that contain 11 double-stranded RNA GSs. The genome is encased within the VP2 (encoded by GS 2), inner VP6 (encoded by GS 6) capsid shell, and the outer glycosylated VP7 (encoded by GS 9) capsid that contains VP4 (encoded by GS 4). VP6 constitutes more than half of the mass of the rotavirus particle (Estes and Kapikian 2007) and contains rotavirus group antigens that are used to sub-classify RVA into subgroups (SG) I, II, I + II, and "non-I, non-II") based on their reactivity to monoclonal antibodies (Greenberg et al. 1983).

Molecular methods have an edge in RVA diagnosis and classification over serological methods due to problems associated with the availability of monoclonal antibodies raised against specific rotavirus antigens. Of late, 16 genotypes (I1-I16) have been determined using a nucleotide sequence-based whole genome classification system (Matthijnssens et al. 2011). There is a correlation 
between the serological and genotype classifications where SG 1 and II corresponds to genotype 2 and 1, respectively (Iturriza-Gómara et al. 2002; Kerin et al. 2007; Matthijnssens et al. 2011).

GS 6 and its encoded VP6 play a major role in RVA detection. VP6 is the primary target antigen for RVA routine diagnostic serological techniques such as enzyme-linked immunosorbent assay (ELISA), immunofluorescence, and immunochromatography (Greenberg et al. 1983; Estes and Kapikian 2007). On the other hand, broadly reactive molecular diagnostic assays such as Reverse Transcriptase-Polymerase Chain Reaction (RT)-PCR protocols targets the ends or internal conserved regions of GS 6 nucleotide sequences by employing hybridization methods which uses sequence specific primers and/or probes (Iturriza-Gómara et al. 2002; Lin et al. 2008). The first molecular assays that were developed in the early 1990s utilised GS 6 nucleotide sequences of rotavirus strains circulating from early 1970s to late 1980s (Grinde et al. 1995). More recently, RT-PCR assays based on GS6 have also been developed using old reference and a few new human rotavirus GS 6 sequences. This resulted in a more reliable scheme for conveying various VP6 genogroups of human rotaviruses (Lin et al. 2008; Matthijnssens et al. 2012).

The availability of many complete GS 6 sequences in the GenBank database will ease the prior challenges of improving, developing and validating rotavirus characterisation methods that utilises VP6 encoding GS (Lin et al. 2008). It is important to continuously evaluate the nucleotide sequences for GS 6 of strains circulating particularly in African countries owing to the wide rotavirus $\mathrm{G}$ and $\mathrm{P}$ genotype variations that have been consistently reported (Mwenda et al. 2010; Kiulia et al. 2014; Seheri et al. 2014; Tsolenyanu et al. 2014). Such studies would inform in advance, potential evolutionary variations that would eventually affect efficiency of VP6 based rotavirus characterisation methods. In this report, the genetic variation of the GS 6 of rotavirus strains collected recently in Pretoria, South Africa, were characterised and compared against those of strains reported from other parts of the world.

\section{Materials and methods}

\section{Ethical consideration and sample selection}

The project was approved by the Medunsa Research Ethics Committee (MREC/P/168/2008; MREC/P/108/2013; PG). Eleven human stool specimens were selected randomly from previously screened ELISA rotavirus-positive samples from children presenting with diarrhoea at Lancet private pathology laboratories, Pretoria in 2008. These samples included two commonly characterised G1P[8], six uncommon G and P genotype combinations (G1P[4] and G2P[6]), and three G9P[8]/G9P [6] strains that have emerged in the last two decades. One rotavirus ELISA rotavirus-positive porcine sample obtained from the archive of the Medical Research Council-Diarrhoeal Pathogens Research Unit (MRC-DPRU) laboratory that could not be assigned G and $\mathrm{P}$ genotypes was also analysed. Nucleotide sequences for human and animal rotavirus strains containing G2, G8, G9 and G12 were selected from our previous studies (Jere et al. 2011, 2012, 2014; Nyaga et al. 2013) to cover frequently characterised strains around this region.

\section{VP7, VP4 and VP6 RT-PCR and genotyping of rotavirus strains} Rotavirus dsRNA was extracted from 10\% fecal supernatant using the QIAamp viral extraction kit by following the manufacturer's instructions (Qiagen, Valencia, CA). Synthesis of cDNA and genotyping of the GS 4 and 9 were performed using RT-PCR as described previously (Góuvea et al. 1990; Gentsch et al. 1992; Iturriza-Gómara et al. 2001; Iturriza-Gómara et al. 2004). In-house designed primer set VP6F (nt 1-20, 5'GGCTTTAAAACGAAGTCTTC3') and VP6R (nt 1336-1356, 5'TGTAGTGAGAGGATGTGACC $\left.3^{\prime}\right)$ was used to amplify the entire GS 6 (1356 bp). In brief, RNA was denatured at $95^{\circ} \mathrm{C}$ for $5 \mathrm{~min}$ followed by reverse transcription at $50^{\circ} \mathrm{C}$ for $30 \mathrm{~min}$. Thirty PCR cycles of $95^{\circ} \mathrm{C}$ for $30 \mathrm{sec}, 50^{\circ} \mathrm{C}$ for $30 \mathrm{sec}$ and $72^{\circ} \mathrm{C}$ for $45 \mathrm{sec}$, followed by an elongation step at $72^{\circ} \mathrm{C}$ for $7 \mathrm{~min}$ was performed. The PCR products were purified with the QIAquick PCR Purification Kit (Qiagen, Valencia, CA) by following the manufacturer's instructions. The PCR amplicon were run on a $1 \%$ Tris-acetate- ethylenediaminetetraacetic acid- agarose gel stained with $1 \%$ ethidium bromide. The GS 6 cDNA amplicons were sequenced using the Sanger ABI 3130xl at Inqaba Biotechnical Industries (Pty) Ltd, Pretoria, South Africa. RotaC [http://rotac.regatools.be/] (Maes et al. 2009) was used to assign genotypes to GS 6 .

\section{Phylogenetic method}

Sequences were aligned using the MUSCLE program within MEGA version 5 (Tamura et al. 2011). Once aligned, the JModelTest 2 program (Posada 2008) was used to identify the optimal evolutionary model that best fitted the sequence datasets. Using corrected Akaike Information Criterion (AICc) the following model; $\mathrm{TN} 93+\mathrm{G}+\mathrm{I}$ was found to best fit the sequence data for the VP6 gene. Using these models, maximum likelihood trees were constructed using MEGA version 5 with 500 bootstrap replicates to estimate branch support. Nucleotide and amino acid distance matrixes were prepared using the $p$-distance algorithm in MEGA version 5 (Tamura et al. 2011).

\section{Results}

The complete sequences of the GS 6 of each selected strain contained $1356 \mathrm{nt}$ and a single ORF from nt 24 to 1214 resulting in deduced protein of 397 amino acid. These were 


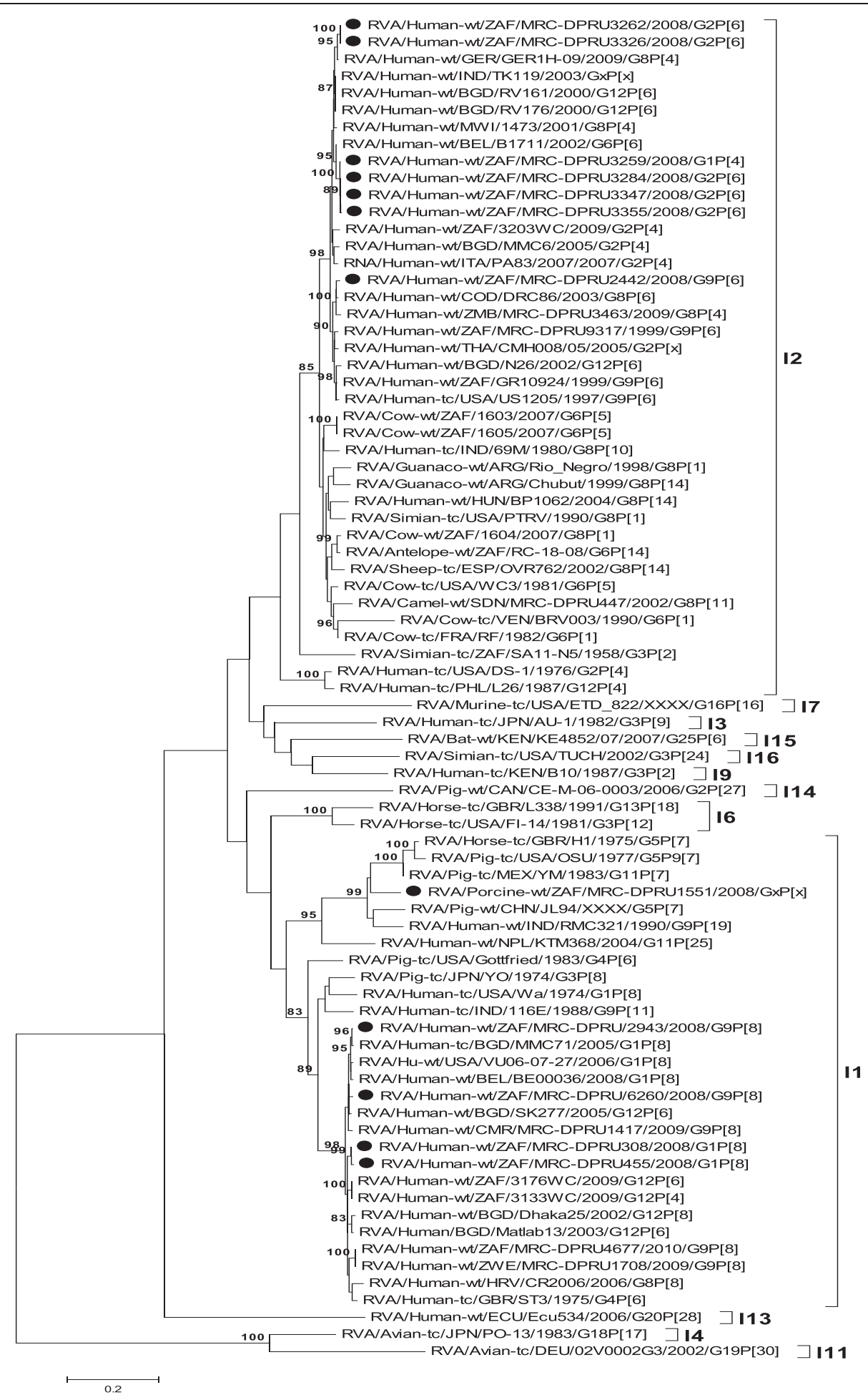

Figure 1 Maximum likelihood phylogenetic trees built in MEGA version 5 with bootstrap statistics as support, show the genetic relationships of nucleotide sequences of VP6 of human and porcine strains from South Africa with known human and animal rotavirus VP6 sequences from the GenBank database. The tree was drawn to scale. Only bootstrap values of 80\% and greater are shown. Bars represent 0.2 substitutions per nucleotide position. South African study strains are indicated by filled circle. 
submitted to the GenBank under accession numbers KC618421-KC618432.

GS 6 of the study strains were assigned either I1 or I2 genotypes as indicated in Additional file 1: Table S1. All the South Africa strains with genotype combination of G1P[8] or G9P [8] were assigned VP6 genotype I1 and they shared a nucleotide (amino acid) identity in the range of 82.8-99.6\% (91.7-99\%), while those with G2P[6] or G1P[4] or G9P[6], were closely related to strains belonging to genotype I2 and shared a nucleotide (amino acid) identity in the range of $79.2-100 \%$ (91.2-100\%). The South African VP6 genotype I1 strains analysed in this study shared a very high nucleotide (amino acid) identity in the range of $81.1-99.7 \%$ (91.4-99.7\%) with both reference human (such as RVA/Human-tc/USA/Wa/1974/G1P[8]) and animal (such as RVA/Pig-tc/USA/OSU/1977/G5P9[7]) genotype I1 VP6 sequences obtained from the GenBank database. On the other hand, South African strains assigned as VP6 genotype I2 also shared a nucleotide (amino acid) identity in the range of $86.6-99 \%$ (96.2-99.7\%) to both reference human (such as RVA/Human-tc/USA/DS-1/ 1976/G2P[4]) and animal (such as RVA/Sheep-tc/ESP/ OVR762/2002/G8P[14]) strains in the same genotype (Additional file 1: Table S1). Phylogenetic analysis divides the South African strains into two separate clusters (Figure 1). Consistent with distance analyses, South African strains in genotype I1 formed a phylogenetic cluster with human rotavirus SGII (Wa-like or genotype I1) strains, while the remaining in genotype I2 clustered with DS1-like or I2 genotype (SGI) GS 6.

Comparison of the amino acid sequences of VP6 proteins of South African strains determined in this study and those from our previous studies, couple with some human and animal rotavirus reference strains reveals several regions that are completely or highly conserved among the South African GS 6 sequences compared to their respective genotypes (Additional file 2). However, South African strains belonging to the I1 or I2 genotypes were closely related to Wa-like or DS-1-like strains, respectively (Additional file 1: Table S1 and Figure 1). Comparison of the group specific antigenic regions of the South African strains to the reference strain RF, shows that the South African VP6 sequences belonging to the VP6 genotype I2 were highly conserved, with only two amino acids changes at positions $239(\mathrm{~T}>\mathrm{N})$ and $261(\mathrm{I}>\mathrm{V})$. On the other hand, South African VP6 sequences belonging to I1 genotypes revealed eight amino acid changes at positions 39(I>V), 45(E>D), 60(N>T), $239(\mathrm{~T}>\mathrm{N}), 248(\mathrm{Y}>\mathrm{F})$, 252(V>I), 261(I>V) and $396(\mathrm{~V}>\mathrm{I})$. Also, the Subgroup I (SGI) and Subgroup II (SGII) residues were highly conserved among South African strains belonging to I2 genotype, while amino acid changes were observed in SGI residues at positions $172(\mathrm{~A}>\mathrm{M}), 305(\mathrm{~A}>\mathrm{N})$ and 310 $(\mathrm{N}>\mathrm{Q})$ of South African strain in I1 genotype.

\section{Discussion and conclusions}

The GS 6 in this study showed significant amounts of genetic variation among strains within the two genotypes. The fact that strains within the I2 genotype were more conserved with less amino acid changes than the I1 strains was supported by the differences in the nucleotide and deduced amino acid identities. For instance, the nucleotide changes for genotype I1 diverged by nt (aa) 3.1 (0.5) and 18.0 (8.6) between themselves and when compared to reference sequences from the GenBank, respectively. Those of I2 diverged by a wider margin, nt (aa) 17.2 (8.3) and 18.0 (8.6) between themselves and when compared to reference sequences from the GenBank, respectively. This could mean that in Pretoria, South Africa, genotype I1 is more prone to genetic changes than genotype I 2 and could potentially result in detection inconsistencies in future. The effect of the observed genetic variations on the host disease presentation warrants further understanding.

In these analyses, the GS 6 of previously analysed local circulating strains were also included (Jere et al. 2011, 2012, 2014; Nyaga et al. 2013). Like any other rotavirus GS, the VP6 encoding GS 6 from these strains could be swiftly evolving at different rates due to antigenic drifts caused by point mutations and was clearly shown by the clustering of strain RVA/Human-wt/ZAF/MRC-DPRU3259/ 2008/G1P[4] with I2 strains. The G1s have previously been reported as only I1 strains (Ghosh and Kobayashi 2011). The nucleotide and amino acid changes reported here were consisted with other studies (Johne et al. 2011; Matthijnssens et al. 2012). Changes occurring at the nucleotide level of VP6-encoding gene over time could cause amino acid sequence variations resulting in changes in VP6 epitopes. This could potentially affect the efficiency of the rotavirus detection methods (Kerin et al. 2007).

The current VP6 detection techniques should work efficiently as the changes observed in the antigenic regions of these South African strains seems not to vary significantly. The limitation of this study is the small sample size albeit including various distinct strains, hence a conclusively determination on how rare or common these changes are occurring in Pretoria, South Africa, cannot be made. Although the sample size was small, the findings were consistent with those reported elsewhere (Kerin et al. 2007; Johne et al. 2011; Matthijnssens et al. 2012). The results suggest that rotavirus investigators should continually consider this diversity and update the primer required for the detection and characterization of the VP6 gene accordingly.

\section{Additional files}

Additional file 1: Table S1. Nucleotide (NT) and amino acid (AA) percentage identities of South Africa VP6 sequences compared to VP6 sequences of human and animal obtained from the GenBank. 
Additional file 2: Comparison of deduced amino acid sequences of VP6 proteins of South African strains to strain RF. Gray areas indicates boundaries of group specific antigenic regions (antigenic site I (32-64), antigenic site II (155-167), antigenic site III (208-274) and antigenic site IV (380-397). Residues 228-240 (Gray box area) indicates hyper variable region, residues 172, 296-300, 305 and 310 (plain box) are Subgroup I (SGI) and residues at position 306 is a Subgroup II (SGII).

\section{Abbreviations}

VP: Viral protein; GS: Genome segment; RVA: Group A rotaviruses; WHO: World Health Organization; RNA: Ribonucleic acid; SG: Subgroup; ELISA: Enzyme linked immunosorbent assay; RT-PCR: Reverse transcriptase polymerase chain reaction; MREC: Medunsa Research Ethics Committee; MRC: Medical research council; DPRU: Diarrhoeal Pathogens Research Unit; cDNA: complementary Deoxyribonucleic acid; kb: Kilobase; AICs: Akaike Information Criterion; nt: Nucleotide; aa: Amino acid.

\section{Competing interests}

The authors declare that they have no competing interests.

\section{Authors' contributions}

$\mathrm{MMN}, \mathrm{MDE}, \mathrm{KCJ}$ and IP made contributions to the conception, design, data analysis and interpretation of all the genetic data including drafting the manuscript. MLS and MJM were involved in project design and critical review of the manuscript. All authors read and approved the final manuscript.

\section{Acknowledgements}

We thank the Medical Research Council of South Africa and Poliomyelitis Research Foundation for financial support (Grant no: 09/43; 13/62 [PhD]), Lancet laboratories for providing the samples and all staff of the MRC/DPRU for their support.

\section{Author details}

'Medical Research Council/Diarrhoeal Pathogens Research Unit, Department of Virology, Medunsa Campus, University of Limpopo/NHLS Dr George Mukhari Tertiary Laboratory, Pretoria, South Africa. ${ }^{2}$ Gastroenteritis and Respiratory Viruses Laboratory Branch, Division of Viral Diseases, NCIRD, CDC, Atlanta, Georgia, USA. ${ }^{3}$ Department of Clinical Infection, Microbiology and Immunology, Institute of Infection and Global Health, University of Liverpool, Liverpool, UK.

\section{Received: 6 December 2013 Accepted: 27 March 2014} Published: 5 April 2014

\section{References}

Estes M, Kapikian A (2007) Rotaviruses. In: Knipe DM, Howley PM, Griffin DE, Lamb RA, Martin MA, Roizmzn B (eds) Fields Virology, 5th edn. Kluver Health/ Lippincott, Williams\&Wilkins, Philadelphia, pp 1917-1974

Gentsch JR, Glass RI, Woods P, Gouvea V, Gorziglia M, Flores J, Das BK, Bhan MK (1992) Identification of group A rotavirus gene 4 types by polymerase chain reaction. J Clin Microbiol 30:1365-1373

Ghosh S, Kobayashi N (2011) Whole-genomic analysis of rotavirus strains: current status and future prospects. Future Microbiol 6:1049-1065

Góuvea V, Glass RI, Woods P, Taniguchi K, Clark HF, Forrester B, Fang ZY (1990) Polymerase chain reaction amplification and typing of rotavirus nucleic acid from stool specimens. J Clin Microbiol 28(2):276-282

Greenberg HB, Valdesuso J, Van Wyke K, Midthun K, Walsh M, McAuliffe V, Wyatt RG, Kalica AR, Flores J, Hoshino Y (1983) Production and preliminary characterization of monoclonal antibodies directed at two surface proteins of rhesus rotavirus. J Virol 47:267-275

Grinde B, Jonassen TO, Ushijima H (1995) Sensitive detection of group A rotaviruses by immunomagnetic separation and reverse transcription-polymerase chain reaction. J Virol Methods 55:327-338

Iturriza-Gómara M, Isherwood B, Desselberger U, Gray J (2001) Reassortment in vivo: driving force for diversity of human rotavirus strains isolated in the United Kingdom between 1995 and 1999. J Virol 75:3696-3705

Iturriza-Gómara M, Wong C, Blome S, Desselberger U, Gray J (2002) Molecular characterization of VP6 genes of human rotavirus isolates: correlation of genogroups with subgroups and evidence of independent segregation. J Virol 76:6596-6601

Iturriza-Gómara M, Kang G, Gray J (2004) Rotavirus genotyping: keeping up with an evolving population of human rotaviruses. J Clin Virol 31:259-265
Jere KC, Mlera L, O'Neill HG, Potgieter AC, Page NA, Seheri ML, Van Dijk AA (2011) Whole genome analyses of African G2, G8, G9, and G12 rotavirus strains using sequence-independent amplification and $454^{\circ}$ pyrosequencing. J Med Virol 83:2018-2042

Jere KC, Mlera L, O'Neill HG, Peenze I, Van Dijk AA (2012) Whole genome sequence analyses of three African bovine rotaviruses reveal that they emerged through multiple reassortment events between rotaviruses from different mammalian species. Vet Microbiol 159:245-250

Jere KC, Esona MD, Ali YH3, Peenze I, Roy S, Bowen MD, Saeed IK, Khalafalla Al, Nyaga MM, Mphahlele J, Steele D, Seheri ML (2014) Novel NSP1 genotype characterised in an African camel G8P[1 1] rotavirus strain. Infect Genet Evol 21:58-66

Johne R, Otto P, Roth B, Löhren U, Belnap D, Reetz J, Trojnar E (2011) Sequence analysis of the VP6-encoding genome segment of avian group F and G rotaviruses. Virology 412:384-391

Kerin TK, Kane EM, Glass Rl, Gentsch JR (2007) Characterization of VP6 genes from rotavirus stains collected in the United States from 1996-2002. Virus Genes 35:489-495

Kiulia NM, Nyaga MM, Seheri ML, Wolfaardt M, Van Zyl WB, Esona MD, Irimu G, Inoti M, Gatinu BW, Njenga PK, Taylor MB, Nyachieo A (2014) Rotavirus G and $P$ types circulating in the eastern region of Kenya: predominance of $G 9$ and emergence of G12 genotypes. Pediatr Infect Dis J 1:85-88

Lin YP, Kao CL, Chang SY, Taniguchi K, Hung PY, Lin HC, Huang LM, Huang HH, Yang JY, Lee CN (2008) Determination of human rotavirus VP6 genogroups I and II by reverse transcription-PCR. Clin Microbiol 46:3330-3337

Maes P, Matthijnssens J, Rahman M, Van Ranst M (2009) RotaC: a web-based tool for the complete genome classification of group A rotaviruses. BMC Microbial 9:238, 238

Mapaseka SL, Dewar JB, van der Merwe L, Geyer A, Tumbo J, Zweygarth M, Bos P, Esona MD, Steele AD, Sommerfelt H (2010) Prospective hospital-based surveillance to estimate rotavirus disease burden in the Gauteng and North West Province of South Africa during 2003-2005. J Infect Dis 202:131-138

Matthijnssens J, Ciarlet M, McDonald SM, Attoui H, Bányai K, Brister JR, Buesa J, Esona MD, Estes MK, Gentsch JR, Iturriza-Gómara M, Johne R, Kirkwood CD, Martella V, Mertens PP, Nakagomi O, Parreño V, Rahman M, Ruggeri FM, Saif L, Santos N, Steyer A, Taniguchi K, Patton JT, Desselberger U, Van Ranst M (2011) Uniformity of rotavirus strain nomenclature proposed by the Rotavirus Classification Working Group (RCWG). Arch Virol 156:1397-1413

Matthijnssens J, Otto PH, Ciarlet M, Desselberger U, Van Ranst M, Johne R (2012) VP6-sequence-based cutoff values as a criterion for rotavirus species demarcation. Arch Virol 157:1177-1182

Mwenda JM, Ntoto KM, Abebe A, Enweronu-Laryea C, Amina I, Mchomvu J, Kisakye A, Mpabalwani EM, Pazvakavambwa I, Armah GE, Seheri LM, Kiulia NM, Page N, Widdowson MA, Steele AD (2010) Burden and epidemiology of rotavirus diarrhea in selected African countries: preliminary results from the African Rotavirus Surveillance Network. J Infect Dis 202:5-11

Posada D (2008) jModelTest: phylogenetic model averaging. Mol Biol Evol 25:1253-1256

Nyaga MM, Jere KC, Peenze I, Mlera L, Van Dijk AA, Seheri ML, Mphahlele MJ (2013) Sequence analysis of the whole genomes of five African human G9 rotavirus strains. Infect Genet Evol 16:62-77

Seheri M, Nemarude L, Peenze I, Netshifhefhe L, Nyaga MM, Ngobeni HG, Maphalala G, Maake LL, Steele AD, Mwenda JM, Mphahlele JM (2014) Update of rotavirus strains circulating in Africa from 2007 through 2011. Pediatr Infect Dis J 1:76-84

Tamura K, Peterson D, Peterson N, Stecher G, Nei M, Kumar S (2011) MEGA5: molecular evolutionary genetics analysis using maximum likelihood, evolutionary distance, and maximum parsimony methods. Mol Biol Evol 28:2731-2739

Tate JE, Burton AH, Boschi-Pinto C, Steele AD, Duque J, Parashar UD (2012) The WHO-coordinated global Rotavirus Surveillance Network. 2008 estimate of worldwide rotavirus-associated mortality in children younger than 5 years before the introduction of universal rotavirus vaccination programmes: a systematic review and meta-analysis. Lancet Infect Dis 12:136-141

Tsolenyanu E, Seheri M, Dagnra A, Djadou E, Tigossou S, Nyaga M, Adjeoda E, Armah G, Mwenda JM, Atakouma Y (2014) Surveillance for rotavirus gastroenteritis in children less than 5 years of age in Togo. Pediatr Infect Dis J 1:14-18

WHO (2012) http://www.who.int/immunization/monitoring_surveillance/burden/ estimates/rotavirus/en/

\section{doi:10.1186/2193-1801-3-179}

Cite this article as: Nyaga et al:: Genetic diversity of rotavirus genome segment 6 (encoding VP6) in Pretoria, South Africa. SpringerPlus $20143: 179$ 